01

\title{
Исследование формирования избыточного свободного объема в тройных стыках границ зерен при кристаллизации на примере никеля
}

\author{
(С) Г.М. Полетаев ${ }^{1}$, Д.В. Новоселова ${ }^{1}$, И.В. Зоря ${ }^{2}$, М.Д. Старостенков ${ }^{1}$ \\ ${ }^{1}$ Алтайский государственный технический университет им. И.И. Ползунова, \\ Барнаул, Россия, \\ ${ }^{2}$ Сибирский государственный индустриальный университет, \\ Новокузнецк, Россия \\ E-mail: gmpoletaev@mail.ru
}

(Поступила в Редакцию 2 марта 2017 г.)

Методом молекулярной динамики проведено исследование формирования избыточного свободного объема в тройных стыках границ зерен при кристаллизации на примере никеля. Показано, что в процессе кристаллизации в тройных стыках избыточный свободный объем образуется преимущественно в результате фиксации объема жидкой фазы при встрече трех фронтов кристаллизации, после затвердевания которая содержит высокую долю свободного объема. В некоторых случаях при концентрировании свободного объема в тройных стыках происходит формирование сравнительно небольшого кристаллического субзерна (от одного до нескольких нанометров в диаметре), имеющего отличную от стыкующихся зерен ориентацию и находящегося в состоянии растяжения.

Исследование выполнено при финансовой поддержке РФФИ в рамках научного проекта № 16-48-190182 p_a.

DOI: $10.21883 /$ FTT.2018.05.45775.062

\section{1. Введение}

Тройной стык зерен представляет собой линейный дефект, вдоль которого сопрягаются три границы зерен. Согласно экспериментальным данным, диффузия в области тройного стыка границ зерен протекает значительно интенсивнее, чем вдоль самих границ [1], вклад тройных стыков в диффузию возрастает по мере уменьшения среднего размера зерна в материале. Кроме того, тройные стыки играют важную роль в процессах, связанных с пластической деформацией, генерацией дислокаций, рекристаллизацией [2]. Тройной стык зачастую характеризуется относительно более „рыхлой“ структурой (даже с включениями аморфной фазы [3]), т.е. с более высоким содержанием свободного объема по сравнению с образующими этот стык границами зерен. Вместе с тем, в работах [4,5], выполненных с помощью компьютерного моделирования, было получено, что диффузионная проницаемость тройного стыка не сильно отличается (или не отличается вовсе) от проницаемости границ зерен. В работах [6,7], выполненных ранее в нашем научном коллективе, был получен аналогичный результат, и, кроме того, было показано, что ни по энергетическим, ни по диффузионным характеристикам, тройной стык не отличается от образующих его границ зерен, и является, по сути, их частью. Указанные противоречия экспериментальных данных и результатов компьютерного моделирования в предыдущих работах мы объясняли существованием в поликристаллах разных типов тройных стыков, отличающихся по структуре и свойствам. Так, согласно работе [8], тройные стыки следует разделять, по крайней мере, на два типа: ненапряженные (или равновесные), не содержащие избыточных дефектов, и напряженные (неравновесные), содержащие избыточные дисклинации, частицы второй фазы (в том числе аморфной) и другие дефекты.

Несмотря на экспериментальное подтверждение существования „структурно правильных“ равновесных тройных стыков, оставался нерешенным вопрос относительно их невысокой доли в поликристаллах, так как, согласно экспериментальным данным, неравновесных стыков, содержащих избыточный свободный объем, значительно больше, чем равновесных $[1,2]$. В работах $[9,10]$ с помощью компьютерного моделирования нами было показано, что наиболее вероятной причиной формирования избыточного свободного объема в стыках при кристаллизации является фиксация объема жидкой фазы при встрече трех фронтов кристаллизации, в результате затвердевания которой в условиях фиксированного объема (вследствие более низкой плотности жидкой фазы по сравнению с твердой) происходит концентрирование избыточного свободного объема в тройном стыке после затвердевания. Образование в стыке дислокационного или дисклинационного комплексов вследствие зернограничного проскальзывания нами представляется второстепенной причиной.

На рис. 1, $a$ схематически изображена структура металла в процессе кристаллизации в момент встречи фронтов кристаллизации от трех разных центров. Скорость движения фронта кристаллизации существенно 

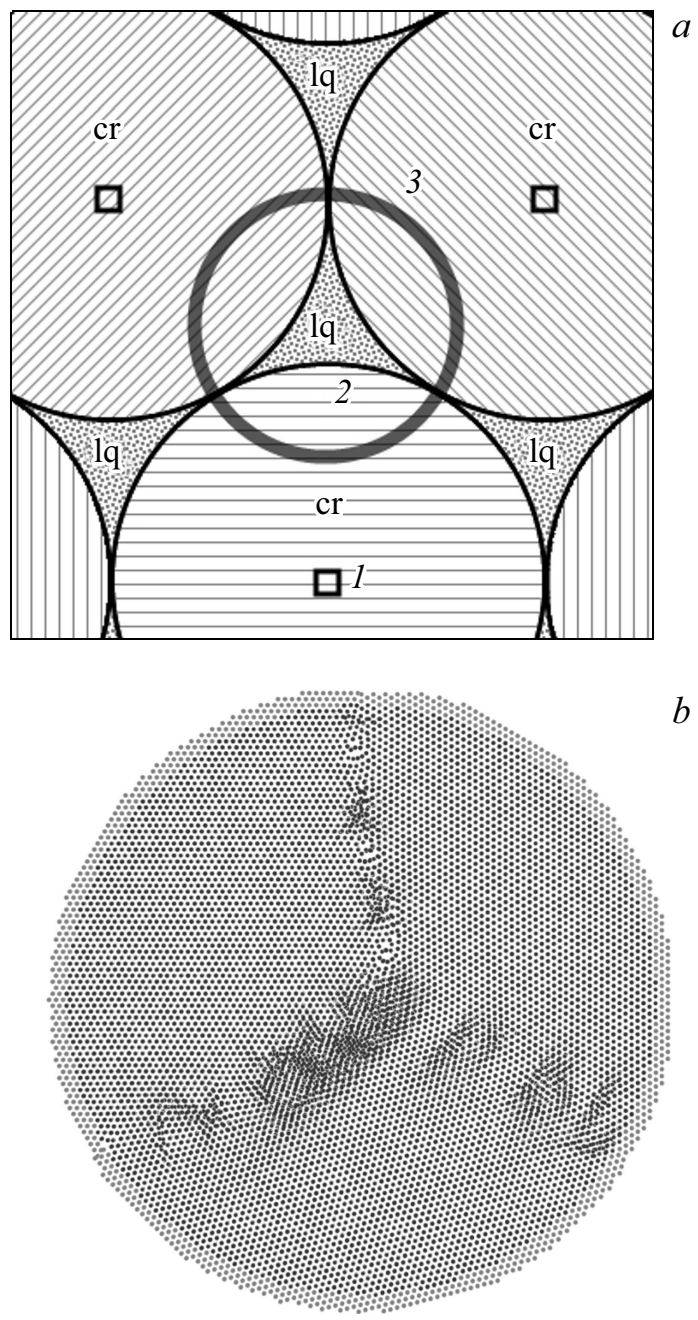

Рис. 1. Формирование избыточного свободного объема в тройном стыке при кристаллизации. $a$ ) схематическое изображение структуры поликристалла в процессе кристаллизации в момент встречи фронтов кристаллизации и фиксации объема жидкой фазы: cr - кристаллическая фаза, lq - жидкая фаза, 1 - центр кристаллизации, 2 - фронт кристаллизации, 3 - границы расчетного блока в молекулярно-динамической модели. $b)$ конечная структура в области тройного стыка границ наклона $\langle 111\rangle$ с углами разориентации $15^{\circ}, 15^{\circ}, 30^{\circ}$ после моделирования кристаллизации при температуре $800 \mathrm{~K}$ в условиях введения в стартовый расплавленный расчетный блок свободного объема (удаления 120 атомов из 30000). Рис. взяты из [10].

ниже скорости звука, поэтому дефекты не „замораживаются“ там, где они есть, а формируются в месте сопряжения кристаллических фаз с разной ориентацией, т.е. в области границ зерен и тройных стыков. При этом именно тройные стыки кристаллизуются в последнюю очередь тем, где встречаются три фронта кристаллизации. При встрече трех фронтов кристаллизации (рис. $1, a)$ плотность оставшейся в области стыка жидкой фазы, которая еще не успела кристаллизоваться, ниже, чем плотность кристаллической фазы. Этот недостаток атомов для формирования „идеального“ тройного стыка приводит к появлению избыточного свободного объема, который рассматривается в процессе кристаллизации преимущественно в тройном стыке. В [10] было показано, что в этом случае концентрация условных вакансий (т. е. доля недостающих атомов по сравнению с равновесными тройными стыками) может достигать в области стыка $2 \%$.

На рис. $1, b$ изображена конечная структура в области тройного стыка границ наклона $\langle 111\rangle$ с углами разориентации $15^{\circ}, 15^{\circ}, 30^{\circ}$ в результате моделирования методом молекулярной динамики кристаллизации никеля при температуре $800 \mathrm{~K}$ в условиях введения в стартовый расплавленный расчетный блок свободного объема (удаления 120 атомов из 30000). На границы цилиндрического расчетного блока накладывались жесткие граничные условия (атомы на боковой поверхности цилиндрического расчетного блока оставались неподвижными в процессе компьютерного моделирования). Жесткие границы в данном случае имитировали фронты кристаллизации от трех центров кристаллизации. Рис. $1, a$ и $b$ взяты из предыдущей нашей работы [10].

Использованная в $[9,10]$ модель, однако, имеет недостатки. Например, она исключает массоперенос вдоль границ зерен в направлении тройного стыка, который может привести к снижению в нем избыточного свободного объема. Для подтверждения сделанного в $[9,10]$ вывода в настоящей работе было проведено исследование кристаллизации металлической пленки. Данная модель позволяла имитировать встречу фронтов кристаллизации от нескольких центров (зародышей кристаллизации). При этом не исключалась возможность диффузионных процессов вдоль границ зерен и выход свободного объема вдоль них на поверхность.

\section{2. Описание модели}

Моделирование проводилось с помощью метода молекулярной динамики. Расчетный блок Ni имел форму пластины (один из размеров расчетного блока задавался сравнительно небольшим - около $1.5-2 \mathrm{~nm}$ ), которая содержала специально введенные кристаллические затравки (зародыши), представляющие собой цилиндрические кристаллические области с неподвижно зафиксированными атомами. Кристаллические зародыши использовались по причине сложности моделирования кристаллизации в молекулярно-динамической модели из жидкого состояния при типичных для этого метода скоростях охлаждения. Дело в том, что для формирования кристаллической структуры из жидкого состояния требуются очень малые, по меркам молекулярной динамики, скорости охлаждения (примерно $10^{12} \mathrm{~K} / \mathrm{s}$ [11]). При обычных скоростях охлаждения, используемых в молекулярной динамике $\left(10^{15}-10^{16} \mathrm{~K} / \mathrm{s}\right)$, при затвердевании из жидкого состояния образуется аморфная структуpa [11]. При создании расчетного блока цилиндрические 
области с кристаллической структурой поворачивались на случайные либо заданные углы вокруг центральной оси цилиндров. Это делалось для того, чтобы по завершении кристаллизации конечные кристаллические зерна имели между собой границы наклона.

На рис. 2 изображены примеры расчетных блоков, содержащих 4 (рис. 2, $a$ ) и 12 (рис. 2, $b$ ) кристаллических затравок. Расчетные блоки содержали 20-30 тысяч атомов. Толщина расчетных блоков вдоль оси $Z$ составляла 4-5 параметров кристаллической решетки (15-20 $)$. Рассматривались расчетные блоки, плоскость $X Y$ которых соответствовала кристаллографическим плоскостям (111) и (100) ГЦК-кристалла. Граничные условия вдоль осей $X$ и $Z$ задавались периодическими, а вдоль $Y-$ свободными, чтобы расчетный блок имел возможность менять объем в процессе кристаллизации и изменения температуры.
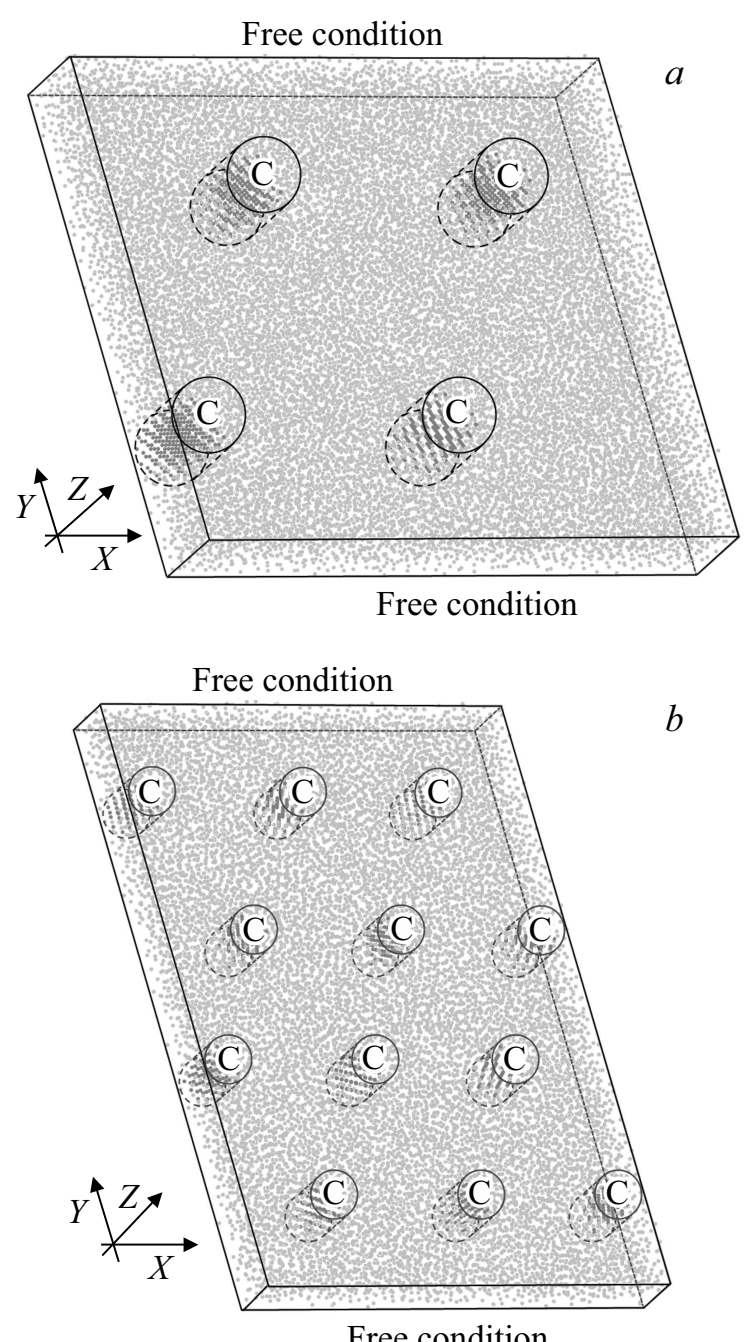

Free condition

Рис. 2. Примеры расчетных блоков для моделирования кристаллизации. Кристаллические затравки (кристаллические зародыши) выделены в виде цилиндрических областей и отмечены буквой „С“: $a$ ) расчетный блок с 4 кристаллическими затравками, $b$ ) с 12 затравками.
Взаимодействия атомов никеля друг с другом описывались многочастичным потенциалом Клери-Розато [12]. Данный потенциал хорошо зарекомендовал себя в ряде расчетов структурно-энергетических характеристик металлов, выполненных методом молекулярной динамики [12,13]. Шаг интегрирования по времени в методе молекулярной динамики был равен $5 \mathrm{fs}$. Температура в модели задавалась через начальные скорости атомов согласно распределению Максвелла.

Кристаллизация в молекулярно-динамической модели проводилась по следующей схеме. Сначала расчетный блок плавился путем нагревания до температуры $3000 \mathrm{~K}$. Затем постепенно охлаждался, при этом температура ступенчато изменялась с 1500 до $800 \mathrm{~K}$. При каждой температуре расчет проводился от нескольких десятков до нескольких сотен пикосекунд. При задании той или иной температуры все межатомные расстояния в расчетном блоке изменялись в соответствии с коэффициентом теплового расширения.

\section{3. Результаты и обсуждение}

На рис. 3 и 4 изображены конечные структуры расчетных блоков, полученных в результате моделирования кристаллизации при наличии 4 (рис. 3) и 12 (рис. 4) кристаллических затравок. Для получения более четкой структуры изображенные расчетные блоки по завершении компьютерного эксперимента были сверхбыстро охлаждены (со скоростью примерно $10^{16} \mathrm{~K} / \mathrm{s}$ ). На рис. $3, b$ и $4, b$ те же расчетные блоки изображены с помощью специальных структурных визуализаторов. Для получения рис. $3, b$ использовался визуализатор среднего расстояния до ближайших атомов. Он дает представление о наличии локального растяжения, а также косвенно о распределении свободного объема. Для каждого атома проводился расчет среднего расстояния до ближайших атомов. Если среднее расстояние незначительно отличалось от расстояния, соответствующего идеальному кристаллу, атом не изображался. В противном случае атом закрашивался в тот или иной оттенок серого цвета.

Для получения рис. $4, b$ использовался визуализатор свободного объема. С помощью черных точек изображены пустоты, не занятые атомами, радиус которых для ГЦК кристалла принимался равным половине параметра кристаллической решетки (минимальный радиус атомов, необходимый для перекрытия октаэдрических пустот в идеальном ГЦК-кристалле). Для визуализации свободного объема пространство в расчетном блоке сканировалось с шагом $0.2 \AA$.

В первую очередь при проведении данных исследований обращалось внимание на то, происходит ли концентрирование свободного объема в процессе кристаллизации в области тройных стыков. Проведенное компьютерное моделирование однозначно показало, что в большинстве случаев свободный объем, действительно, 

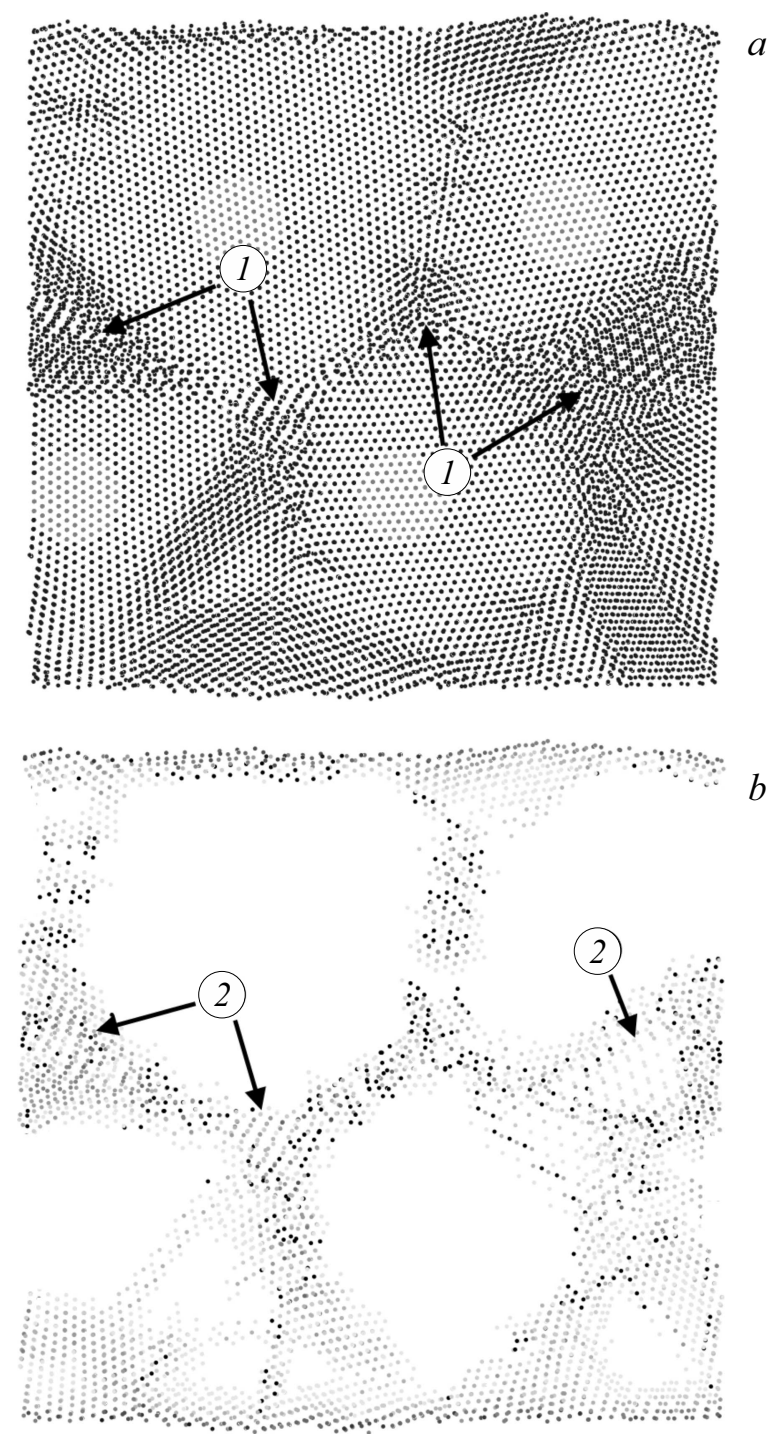

Рис. 3. Изображение расчетного блока в плоскости $X Y(a)$ и соответствующая визуализация средних расстояний до ближайших атомов $(b)$ при наличии 4 кристаллических затравок после моделирования кристаллизации в течение 2000 ps. Плоскость $X Y$ соответствует ориентации (111). 1 - концентрирование и запирание избыточного свободного объема, 2 кристаллические субзерна в растянутом состоянии в тройных стыках.

запирается в области тройных стыков, причем в бо́льшей степени, чем в области границ зерен. На приведенных рисунках цифрой 1 отмечены примеры такого формирования свободного объема. Следует отметить, что свободный объем в процессе диффузии может частично уходить вдоль границ зерен к свободной поверхности. Но это, очевидно, возможно только на сравнительно небольшом расстоянии от нее.

При изучении полученных структур с помощью визуализатора средних расстояний до ближайших атомов было замечено появление свободного объема в некоторых тройных стыках: в стыках иногда наблюда- лось формирование небольших субзерен (от одного до нескольких нанометров в диаметре), имеющих отличную от стыкующихся зерен ориентацию и находящихся в растянутом состоянии. На рис. $3, b$ такие субзерна отмечены цифрой 2.

Несмотря на то, что модель создавалась таким образом, чтобы образовывались только границы наклона, в полученных поликристаллах, особенно в случае ориентации $X Y$ (111), наблюдались границы кручения и
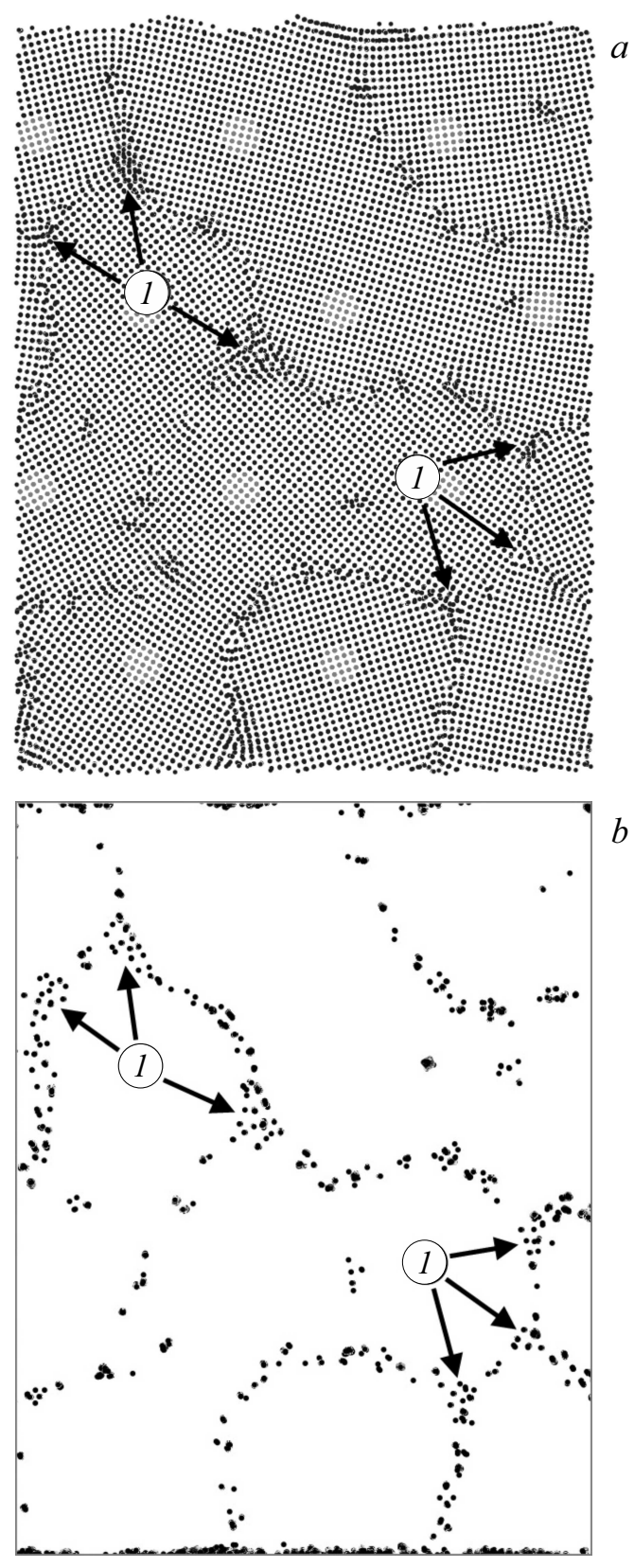

Рис. 4. Изображение расчетного блока в плоскости $X Y(a)$ и соответствующая картина распределения свободного объема $(b)$ при наличии 12 кристаллических затравок после моделирования кристаллизации в течение 200 ps. Плоскость $X Y$ соответствует ориентации (100). 1 - концентрирование избыточного свободного объема. 
смешанного типа. Еще одним интересным результатом проведенных исследований являлось существенное отличие скорости кристаллизации в расчетных блоках, имеющих разную ориентацию: блоки с ориентацией $X Y$ (100) (с соответствующими кристаллическими затравками) кристаллизовались быстрее, чем с ориентацией (111).

\section{4. Заключение}

С помощью молекулярно-динамического моделирования кристаллизации при условии наличия нескольких кристаллических зародышей на примере никеля показано, что избыточный свободный объем формируется в процессе кристаллизации в тройных стыках границ зерен преимущественно в результате фиксации объема жидкой фазы при встрече трех фронтов кристаллизации. Затвердевание структуры вблизи тройных стыков происходит в последнюю очередь, и недостаток плотности при затвердевании структуры в стыке приводит к образованию в них избыточного свободного объема.

В некоторых случаях при наличии свободного объема в тройных стыках происходит формирование сравнительно небольшого кристаллического субзерна (от одного до нескольких нанометров в диаметре), имеющего отличную от стыкующихся зерен ориентацию и находящегося в состоянии растяжения.

\section{Список литературы}

[1] G. Palumbo, K.T. Aust. Scripta Metallurgica Mater. 24, 1771 (1990).

[2] М.Ю. Гуткин, И.А. Овидько. Успехи механики 2, 68 (2003).

[3] P. Rodriguez, D. Sundararaman, R. Divakar, V.S. Raghunathan. Chem. Sustainable Development 8, 69 (2000).

[4] T. Frolov, Y. Mishin. Phys. Rev. B 79, 174110 (2009).

[5] A.G. Lipnitskii, I.V. Nelasov, Yu.R. Kolobov. Defect Diffusion Forum 309-310, 45 (2011).

[6] Г.М. Полетаев, Д.В. Дмитриенко, М.Д. Старостенков. Фундамент. проблемы соврем. материаловедения 9, 344 (2012).

[7] Г.М. Полетаев, Д.В. Дмитриенко, В.В. Дябденков, В.Р. Микрюков, М.Д. Старостенков. ФТТ 55, 1804 (2013).

[8] Э.В. Козлов, Н.А. Конева, Н.А. Попова. Физическая мезомеханика 12, 93 (2009).

[9] G.M. Poletaev, D.V. Novoselova, V.M. Kaygorodova. Solid State Phenomena 249, 3 (2016).

[10] G. Poletaev, D. Novoselova, V. Kaygorodova, M. Starostenkov. AIP Conf. Proc. 1698, 040005 (2016).

[11] G.M. Poletaev, V.Yu. Krasnov, M.D. Starostenkov, N.N. Medvedev. J. Phys. Conf. Ser. 98, 042011 (2008).

[12] F. Cleri, V. Rosato. Phys. Rev. B 48, 22 (1993).

[13] Г.М. Полетаев, М.Д. Старостенков. ФТТ 52, 1075 (2010). 